\title{
A Rare Case of Chronic Alcoholism Related Marchiafava-Bignami Disease
}

\author{
Kanchana Seneviratne ${ }^{\mathrm{a}, \mathrm{b}}$, Sukran Alten ${ }^{\mathrm{a}}$, Mark Farrugia ${ }^{\mathrm{a}}$
}

\begin{abstract}
Marchiafava Bignami disease (MBD) is a rare demyelinating disorder, mainly affecting the corpus callosum. This is a case of a 53 year old genltman with chronic alcoholism who presents with neurological symptoms. Diagnosis is now much easier with MRI imaging. There have only been about 250 patients with MBD noted to be reported in the literature.
\end{abstract}

Keywords: Marchiafava-Bignami disease; Alcoholism; Diagnosis

\section{Introduction}

Marchiafava Bignami disease (MBD) is a rare demyelinating disorder, mainly affecting the corpus callosum. It was first described by two Italian pathologists: Ettore Machiafava and Amico Bignami. It is associated with chronic alcohol abuse. We present a case of this rare Marchiafava Bignami disease in a patient presenting as a fall.

\section{Case Report}

A 53-year old Caucasian man with an alcohol consumption on average of 35 units per week, mainly lager, was admitted following a fall. On admission he appeared unkempt, confused, and had impaired memory. His carer expressed that he was usually confused and that he was likely to have been intoxicated before the fall. There was a one month

Manuscript accepted for publication September 5, 2011

${ }^{a}$ Newham University Hospital NHS Trust, London, UK

${ }^{b}$ Corresponding author: Kanchana Seneviratne.

Email: kanchanaseneviratne@doctors.org.uk

doi:10.4021/jnr57e history of unsteadiness on his feet. Physical examination revealed a malnourished individual and Mini-mental state examination showed short and long term memory deficits. On neurological examination there was reduced coordination, brisk reflexes in all limbs, an ataxic gait, dysdiadokokinesia, dysarthria and an intention tremor. Laboratory results revealed gamma glutamyl transpeptidase 60 (10-49), bilirubin 11 (5-21), alkaline phosphatise 277 (80-300), and alanine transaminase 38 (0-45). His tremors were initially assumed to be secondary to his alcohol withdrawal and were expected to reduce following the detoxification programme which was initiated. Despite the treatment however, the symptoms and signs of tremor, dysarthria and ataxia persisted. Magnetic resonance imaging (MRI) of his brain showed dilatation of the ventricles with cerebral atrophy. Confluent white matter signal abnormalities were present in the periventricular regions (Fig. 1, 2). In the posterior fossa and superior cerebellar vermis similar changes were noted and in the pons, areas of increased signal in the T2-weighted images were observed (Fig. 3, 4). One of the most striking abnormalities, however, was the demyelination of the corpus callosum. The presence of these findings suggested a diagnosis of Marchiafava Bignami. The patient was successfully weaned off alcohol following a detoxification

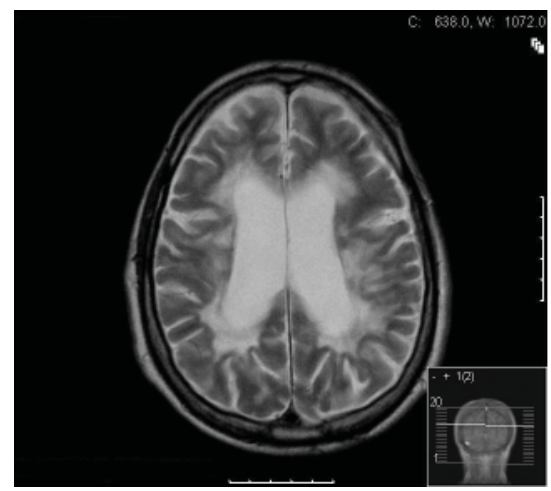

Figure 1. Axial T2W section at level of the lateral ventricles showing moderately dilated symmetrical ventricles with extensive abnormal high signal in the periventricular and deep white matter. The cortical structures appear relatively normal. 


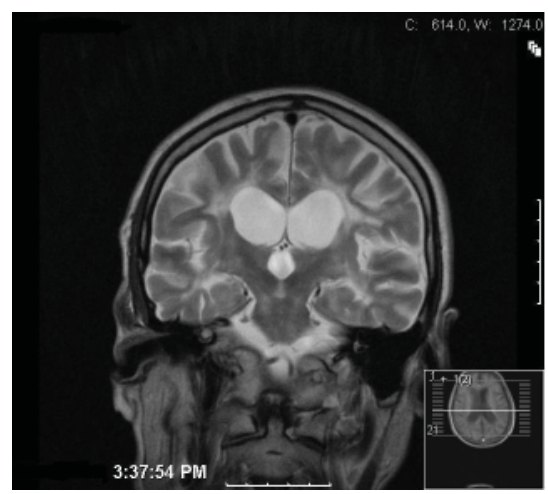

Figure 2. Coronal T2W section at mid parietal level showing the extensive bilateral white matter abnormality consistent with acute demyelination. There is significant thinning of the body of the corpus callosum.

programme with Pabrinex (Intravenous thiamine B1) and chlordiazepoxide. He was then treated with oral thiamine and vitamin B and discharged to a nursing home with improved gait and balance.

\section{Discussion}

The Italian pathologists Marchiafava and Bignami first described this disease following post-mortem brain analysis of three alcoholics (red wine drinkers) who presented with status epilepticus and coma in 1903. About 250 patients with MBD have been reported in several countries [1]. However it is felt that MBD goes under-reported and under-diagnosed and that its incidence is higher than that reported. This disease is due to demyelination of the corpus callosum and can also involve the cerebellar peduncles and putamen.

Diagnosis is made on the basis of clinical findings in

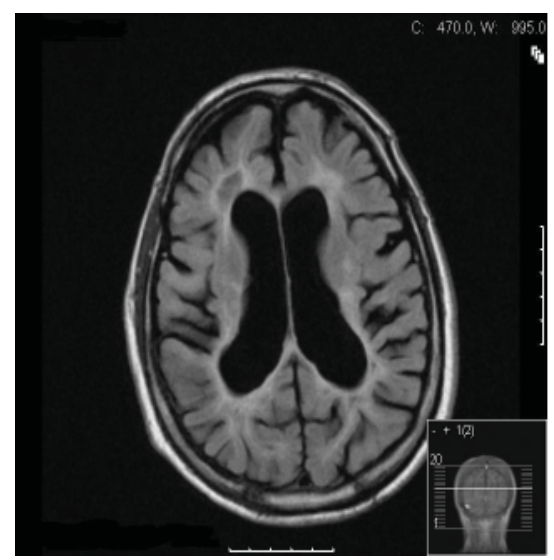

Figure 3. Axial STIR image showing extensive symmetrical periventricular and deep white matter signal abnormality consistent with acute demyelination. Note the generalised alcohol related atrophic changes.

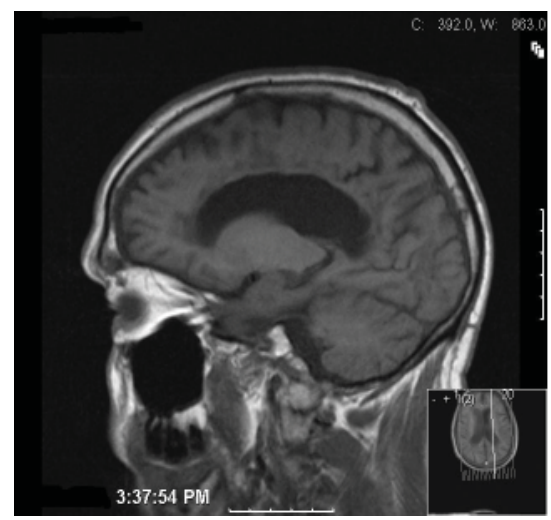

Figure 4. T1W mid sagittal image showing the atrophy of the corpus callosum with altered signal within the corpus callosum indicative of demyelination and chronic changes.

combination with radiological imaging features. Diagnosis is now much easier with MRI imaging. The presenting features are non specific and include confusion, disorientation, psychotic and emotional symptoms, seizures, hemiparesis, dysarthria, ataxia, coma and death [2]. Most patients presenting with the acute type of MBD will go into coma and eventually die, although as in this case report, some survive.

In this case, the diagnosis was made on the basis of chronic alcohol abuse associated with the unresolving neurological symptoms and the MRI findings. The differential diagnosis in such a case would be Wernicke's encephalopathy, Korsakoff syndrome and pontine myelinolysis. MRI imaging is most useful in differentiating MBD from the above differentials [3, 4]. The pathophysiology of MBD remains unclear. The involvement of the entire corpus callosum appears to be associated with a poorer neurological diagnosis. It is important to consider Marchiafava Bignami disease when non specific neurological symptoms with or without encephalopathy are present in an alcoholic. Early imaging with MRI should be considered in these cases.

\section{References}

1. Shintani S and Shigaii T. Marchiafava Bignami disease with only slowly progressive cognitive impairment. Journal of rural medicine 2007; 1: 62-66.

2. Arbelaez A, Pajon A, Castillo M. Acute MarchiafavaBignami disease: MR findings in two patients. AJNR Am J Neuroradiol. 2003;24(10):1955-1957.

3. Hlaihel C, Gonnaud PM, Champin S, Rousset H, TranMinh VA, Cotton F. Diffusion-weighted magnetic resonance imaging in Marchiafava-Bignami disease: followup studies. Neuroradiology. 2005;47(7):520-524.

4. Gerlach A, Oehm E, Wattchow J, Ziyeh S, Glocker FX, Els T. Use of high-dose cortisone in a patient with Marchiafava-Bignami disease. J Neurol. 2003;250(6):758-760. 